\title{
Broadband single-mode microfiber coupler for OCT
}

\author{
Yongmin Jung, Gilberto Brambilla, and David J. Richardson \\ Optoelectronics Research Centre, University of Southampton, Southampton, SO17 1BJ, UK \\ ymj@orc.soton.ac.uk
}

\begin{abstract}
We present a bi-conical microfiber coupler (MFC) with a specifically designed transition region as an efficient tool to filter higher-order modes in multimode waveguides. Higher-order modes are effectively suppressed by controlling the transition taper profile and the diameter of the optical microfiber. As a practical example, single-mode operation over a broad spectral window (400 1700 nm) was demonstrated with a $1.1 \mu \mathrm{m}$ MFC made from conventional telecom optical fibres.

(C)2008 Optical Society of America

OCIS codes: (230.1150) All-optical devices; (060.2340) Fiber optics components; (220.4000) Microstructure fabrication;
\end{abstract}

\section{Introduction}

Stable single-mode operation in photonic waveguides is essential for modern science and technology. The phenomenal growth of the high performance fiber lasers, sensors, photolithography, and optical coherent tomography systems [1] has increased the demand for short wavelength and wide-range single-mode operation. How to achieve stable, low-loss and large-core single-mode beam operation in short wavelengths and over a wide range of wavelengths is actually a major technological challenge. In a recent work [2], the authors successfully demonstrated sub-wavelength optical microwires as an efficient tool for higher-order mode filtering in multimode waveguides, creating useful endlessly single mode operation in conventional optical fibers. Here we present a novel fused-type microfiber coupler for broadband single-mode operation in a few-mode fiber. As a practical example, single-mode operation of a standard telecom optical fiber over a broad spectral window (400 1700 nm) was demonstrated with a $1.1 \mu \mathrm{m}$-diameter microfiber.

\section{Operating principle and fused microfiber coupler fabrication}

Fig. 1 represents an idealized microfiber coupler for higher-order mode filtering, which is composed of two conical transition tapers and a central uniform waist region. The structure is essentially the same as previously wellestablished fused-type optical fiber coupler, except for the extremely small waist diameter $(\sim 1 \mu \mathrm{m})$. As described in ref [2], higher-order modes can be effectively suppressed by controlling the MFC diameter in the uniform waist region, which suppresses propagation of higher-order modes along the entire length of the MFC and constrains the number of guided modes. By designing an appropriate non-adiabatic transition taper it is possible to convert highorder core modes to higher-order cladding modes or radiation modes which are not supported by the MFC. The different mode evolution (adiabatic for fundamental mode and non-adiabatic for higher-order modes) allows only the transverse single mode to propagate along the waveguide, which establishes a single-mode operation for a

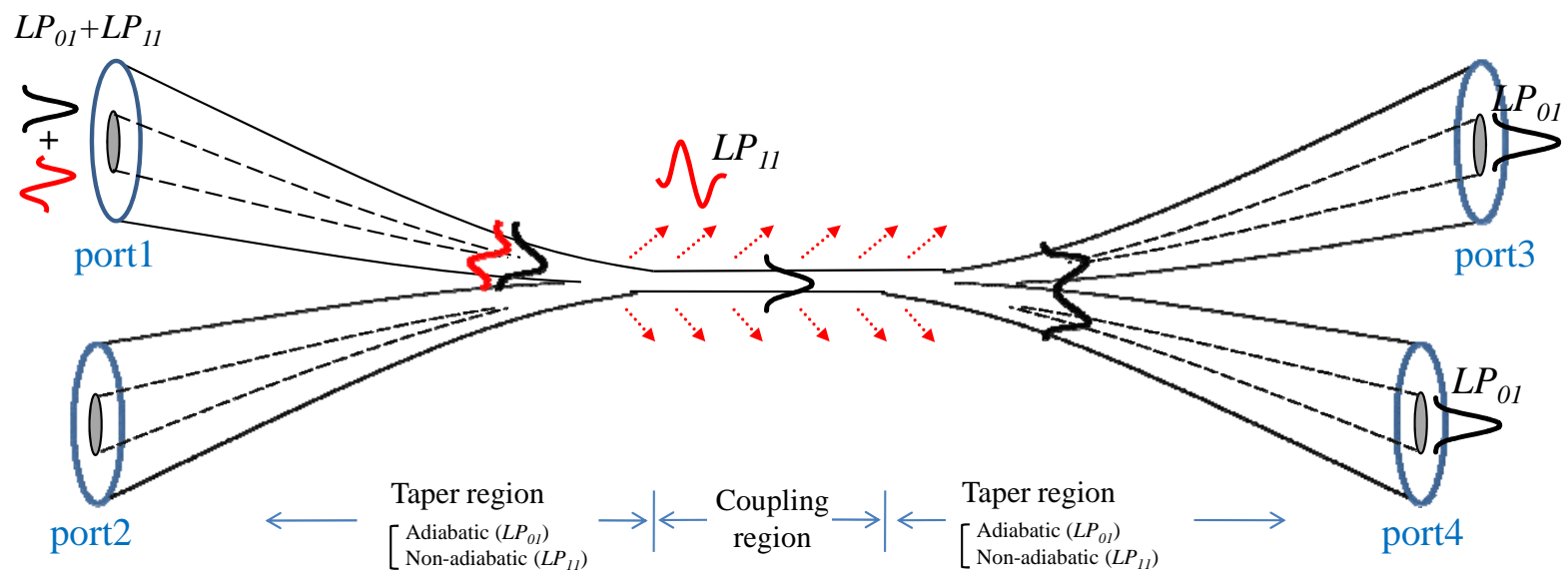

Fig. 1 Schematic diagram of the microfiber coupler (MFC) 


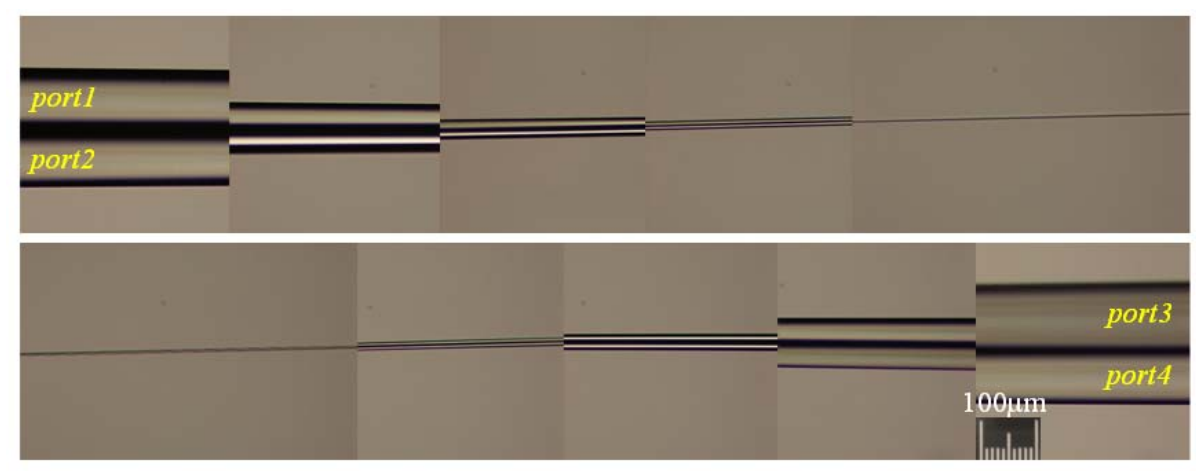

Fig. 2 Microscopic side view of the fibers along the microtaper.

conventional fiber over an extremely wide range of wavelengths. Low-loss MFCs were manufactured with the aid of well-established single-stage conventional "flame-brushing” technique [3, 4]. A standard telecom optical fiber (Corning SMF-28) was selected as a simple example of multimode operation at short wavelengths. The profile of the conical transition tapers was approximated by a decreasing exponential function and was achieved with an appropriate control of the translation stages movement. Fig. 2 shows microscopic side view of the fibers along the microtaper.

\section{Transmission characteristics and far-field patterns}

To investigate the modal guidance, in-situ transmission spectra of the MFCs were recorded for various outer diameters during the fabrication process technique. Fig. 3 shows the spectral output of a telecom optical fiber for different diameters in the uniform waist region. As the waist diameter decreased from 125 to $70 \mu \mathrm{m}$, intermodal interference appears in the multimode spectral region $(<1250 \mathrm{~nm})$, while no perturbation occurs in the single-mode operation range $(1250 \sim 1700 \mathrm{~nm})$. When the outer diameter reaches $20 \mu \mathrm{m}$ the optical power in the output port $P_{3}$ (Fig. 3, left) decrease, and output port $P_{4}$ (Fig. 3, right) increases as the fused time increased. Also the interferometric optical power beating between modes created in the coupling region was observed but their strength decreased, when the waist diameter was further decreased from 20 to $1.1 \mu \mathrm{m}$. Note that for the $1.1 \mu \mathrm{m}$ MFC there is no higherorder mode cut-off and the optical loss is negligible (excess loss $<0.1 \mathrm{~dB}$ at $\lambda=1.55 \mu \mathrm{m}$ ). Fig. $4(\mathrm{a})$ shows the spectral response of two output ports of the fabricated 1.1 $\mu \mathrm{m} \mathrm{MFC} \mathrm{at} \mathrm{a} \mathrm{50:50} \mathrm{coupling} \mathrm{ratio.} \mathrm{It} \mathrm{is} \mathrm{noted} \mathrm{that} \mathrm{output} \mathrm{ports}$ have very uniform spectral characteristics over a wide wavelength range, from 400 to $1700 \mathrm{~nm}$, which can make the device suitable for a wideband power splitter. Fig. 4(b) shows a normalized output power variation from both arms as a function of the elongation time at a wavelength of $1.55 \mu \mathrm{m}$. After the pulling time of 100s, MFC began the mode coupling cycle between two arms; when the elongation time reached around 500s, splitting of the output power into equal halves was achieved to result in a 50:50 coupler. Therefore, it appears that the optimum MFC diameter for efficient higher-order filtering is about $1.1 \mu \mathrm{m}$. Generally, the single-mode operation bandwidth is limited at short wavelengths by a higher-order mode cut-off ( $\lambda_{c_{-}} L P_{11}, \lambda_{c-} L P_{21}$ and $\lambda_{c_{-}} L P_{02}$ in standard optical fibers). However, by applying efficient mode filtering scheme based on sub-wavelength waveguide, a broad range of single-mode optical window (400 1700nm) was successfully realized with minimal loss.
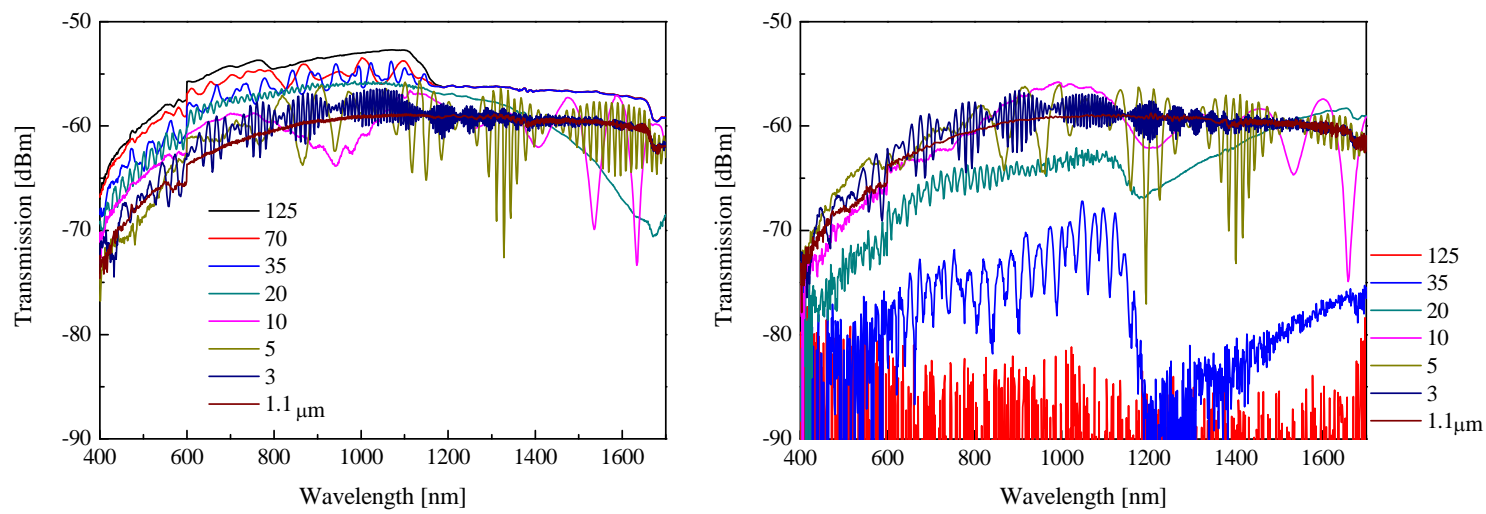

Fig. 3 Spectral response of microfiber coupler: Transmission spectra of the MFC in port 3 (left) and port4 (right) for different MFC outer diameters. 


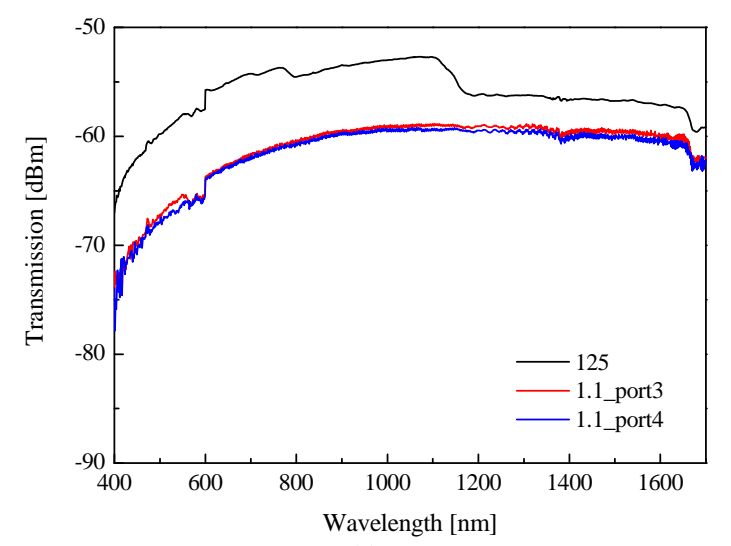

(c)

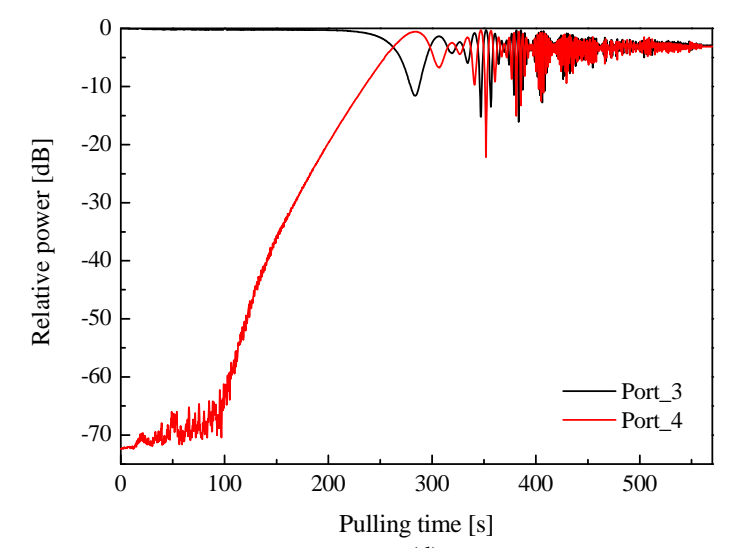

(d)

Fig. 4 (a)Comparison between the transmission spectra of a standard fiber (Corning SMF-28) without (in black) and with (in red, blue) a $1.1 \mu \mathrm{m}$ MFC. (b) Relative coupled power at $\lambda=1.55 \mu \mathrm{m}$ as a function of pulling time

To confirm robust single-mode guidance, the far-field pattern was imaged with a 50× microscopic lens and a CCD camera captured the image at the wavelength of $632.8 \mathrm{~nm}$. When coherent light is guided into a multimode fiber, interference between guided modes travelling within the fiber results in the degradation of the laser beam quality at the waveguide output. In fact, the interference pattern is extremely sensitive to external perturbations such as bending. As predicted, the conventional fiber suffers severe modal interference when applying some bends as shown in Figs. 5(a). However, when an MFC is inserted, the fiber coupler outputs showed high-quality single-mode beams which were not disturbed by external bends (Figs. 5b and 5c). To test the stability of the single mode operation external perturbations such as bends and multi-point splices were applied: no form of optical degradation was detected in the mode profile and/or in the transmission spectrum.

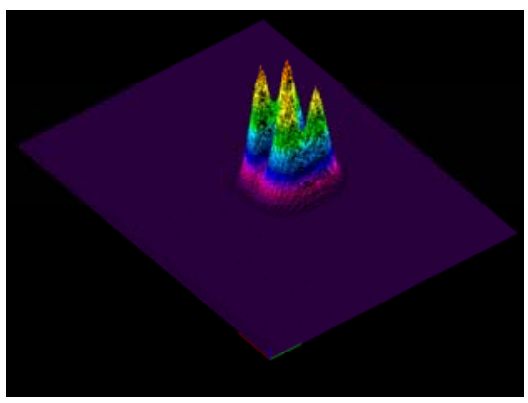

(a)

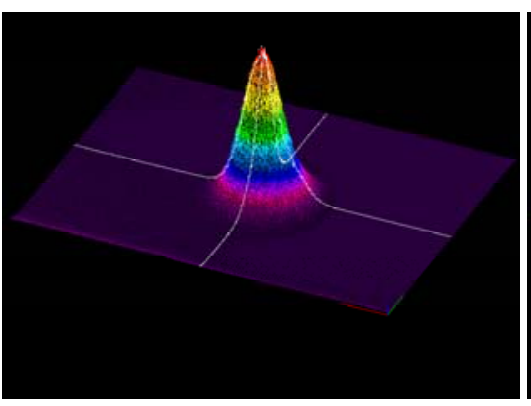

(b)

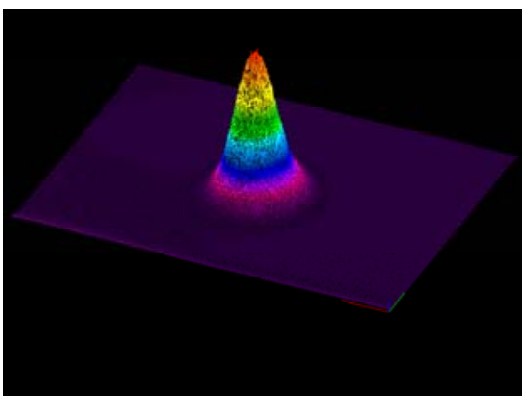

(c)

Fig. 5 Far-field imaging of MFC in the input (a) and the output port (b:port3, c:port4).

\section{Conclusion}

We have demonstrated and validated a novel microfiber coupler for broadband single-mode operation of multimode waveguide. Higher-order modes are effectively suppressed by controlling the transition taper profile and the diameter of the sub-wavelength optical wire. As a practical example, single-mode operation of a standard telecom optical fiber over a broad spectral window (400 1700nm) was demonstrated with a 1.1 $\mu$ m MFC.

The authors thank the Engineering and Physical Sciences Research Council UK (EPSRC) for financial support; GB gratefully acknowledges the Royal Society (London, UK) for his Research Fellowship; the work was partially supported by the Korea Research Foundation Grant funded by the Korean Government (KRF-2008-357-C00040)

\section{References}

[1] K. Okamoto, Fundamentals of Optical Waveguides (Elsevier Academic, London, 2006).

[2] Y. Jung, et. al., "Broadband single-mode operation of standard optical fibers by using a sub-wavelength optical wire filter,” Opt. Express 16, 14661 (2007).

[3] G. Brambilla, V. Finazzi, and D. J. Richardson, “Ultra-low-loss optical fiber nanotapers,” Opt. Express 12, 2258-2263 (2004).

[4] T. A. Birks, and Y. W. Li, “The shape of fiber tapers,” J. Lightwave Technol. 10, 432-438 (1992). 